\title{
B-Vitamin and Choline Supplementation Changes the Ischemic Brain
}

\author{
Prerana J. Keerthi ${ }^{1 *}$ and Nafisa M. Jadavji ${ }^{1}$
}

Recovery following stroke is dependent on several factors, including neuroplasticity. Dietary supplementation with folic acid impacts neuroplasticity. Folic acid is a B-vitamin and is a major component of one-carbon metabolism. The aim of this study is to determine the impact of one-carbon supplementation on neuroplasticity after ischemic stroke using a mouse model. Neuroplasticity was assessed by comparing levels of brain-derived neurotrophic factor (BDNF) and growth differentiation factor 11 (GDF-11) in a mouse model of ischemia. The supplemented diet (SD) contained higher levels of folic acid, riboflavin, vitamin B12, and choline. After ischemic damage, within the damage site, we report that SD mice had increased levels of BDNF compared to control mice. Results also indicated a trend for increased GDF-11 in SD animals; however, these results were not statistically significant. Combined with previous results, this study suggests that supplementation with folic acid, vitamin B12, riboflavin, and choline may promote post-stroke neuroplasticity through increasing levels of BDNF. Increased levels of BDNF may be attributed to the protein kinase A (PKA) mechanism. Future research could focus on optimizing neuroplastic effects by altering concentrations and determining the time frame during which treatment is most effective. Additionally, treatment efficacy with vitamins and nutrients could be assessed in stroke-affected patients. By understanding the role of these vitamins in post-stroke plasticity, novel and safe therapies may be applied to affected patients.

\section{INTRODUCTION}

Stroke is the second leading cause of death worldwide and third leading cause of death in the industrialized world, posing a great burden to health care systems (Corbett et al., 2015; Dirnagl et al., 1999). Ischemic stroke is characterized by a reduction in cerebral blood flow resulting in poor glucose and oxygen delivery to the brain, leading to neuronal and astrocyte cell death (Dirnagl et al., 1999; Table 1).

New approaches for reducing the burden of stroke focus on promoting post-stroke plasticity. Neural plasticity refers to the brain's ability to adapt to new circumstances, such as acquiring a learning a different skill (e.g. riding a bicycle). Neuroimaging studies in human patients have demonstrated neural plasticity during post-stroke recovery by uncovering functional changes in behavior (Hara, 2015). Post-stroke neuroplasticity is thought to occur due to changes in synaptic activity, gene expression, and cell signaling (Hara, 2015). An important marker for neural plasticity is brain-derived neurotrophic factor (BDNF) (Cohen-Cory et al., 2010). Studies have shown that neurons made to produce additional BDNF are protected from apoptosis (Zhang et al., 2011).

\footnotetext{
${ }^{1}$ Department of Neuroscience, Carleton University, Ottawa, Canada K1T 5B6
}

*To whom correspondence should be addressed: pkeerthi11@gmail.com

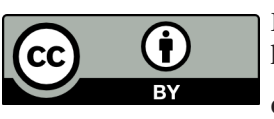

Except where otherwise noted, this work is licensed under https://creativecommons.org/licenses/by/4.0 doi:10.22186/jyi.36.4.44-49
Administration of BDNF in rats has also been reported to reduce the expression of tumor necrosis factor alpha (TNF- $\alpha$ ), an inflammatory molecule, while increasing levels of anti-inflammatory cytokines. This suggests that BDNF may reduce ischemic damage by reducing inflammation (Jiang et al., 2011). Finally, BDNF can inhibit neurotoxicity induced by glutamate in cultured cortical neurons by accumulating in dendrites to activate the tyrosine ki-

Table 1. Definitions of technical terms used in the current study.

\begin{tabular}{|c|c|}
\hline Term & Definition \\
\hline Astrocyte & $\begin{array}{l}\text { Glial cells that support the blood-brain barrier } \\
\text { and provide nutrients to neurons }\end{array}$ \\
\hline $\begin{array}{l}\text { Tumor Necrosis Fac- } \\
\text { tor Alpha }(\mathrm{TNF}-\alpha)\end{array}$ & $\begin{array}{l}\text { Inflammatory molecule that exacerbates isch- } \\
\text { emic injury }\end{array}$ \\
\hline Excitotoxic cell death & $\begin{array}{l}\text { Neuronal death induced by overstimulation of } \\
\text { NMDA receptors }\end{array}$ \\
\hline $\begin{array}{l}\text { Tyrosine kinase } \\
\text { receptor B (TrkB) }\end{array}$ & $\begin{array}{l}\text { Family of receptors that respond to neuro- } \\
\text { trophins }\end{array}$ \\
\hline $\begin{array}{l}\text { Transforming Growth } \\
\text { Factor beta (TGF- } \beta \text { ) }\end{array}$ & $\begin{array}{l}\text { Cytokine that controls cell proliferation, differ- } \\
\text { entiation, and apoptosis }\end{array}$ \\
\hline Homocysteine & $\begin{array}{l}\text { Non-protein amino acid synthesized from me- } \\
\text { thionine through removal of a methyl group }\end{array}$ \\
\hline $\begin{array}{l}\text { Protein Kinase A } \\
\text { (PKA) }\end{array}$ & $\begin{array}{l}\text { Enzyme family responsible for cellular re- } \\
\text { sponses from the cAMP second messenger } \\
\text { pathway }\end{array}$ \\
\hline $\begin{array}{l}\text { Nerve Growth Factor } \\
\text { (NGF) }\end{array}$ & $\begin{array}{l}\text { Family of growth factors responsible for } \\
\text { growth, survival, and neuronal plasticity. }\end{array}$ \\
\hline
\end{tabular}


nase receptor B (TrkB), protecting neurons from excitotoxic cell death as a result of stroke (Chen et al., 2013; Crozier et al., 2008).

Growth differentiation factor 11 (GDF-11) is a member of the transforming growth factor beta (TGF- $\beta$ ) superfamily of molecules, which has recently been reported to reduce age-related changes in muscles and the brain (Brun and Rudnicki, 2015; Horbelt et al., 2012; Katsimpardi et al., 2014; M. Zhang et al., 2018). Reduced levels of GDF-11 have also been reported in the middle cerebral artery occlusion (MCAO) mouse model of stroke, although the role of GDF-11 in ischemic stroke is not well defined (Chauhan et al., 2016).

Environmental factors such as diet also have a large impact on brain plasticity (Murphy et al., 2014). One potential therapy for increasing post-stroke plasticity is nutrition. Specifically, the use of folic acid, a B-vitamin, which has been reported to reduce the risk of stroke in adults with hypertension (Huo et al., 2015). Folic acid decreases level of homocysteine, which has been linked to increased risk of stroke (Castro et al., 2006). Elevated levels of homocysteine may alter synaptic function by activating glutamate receptors and promoting excitotoxicity (Lipton et al., 1997). Folic acid may combat ischemic damage by decreasing levels of homocysteine to limit its oxidative stress and excitotoxicity.

Another important function of folic acid is the generation of methyl groups for DNA methylation. It has been reported that DNA methylation can control BDNF expression during forebrain development in mice (Dennis and Levitt, 2005; Iskandar et al., 2010). Animals deficient in vitamin B12 have reduced levels of neurotrophic factors, such as BDNF (Sable et al., 2011). In addition, B-vitamins have a protective effect (Selhub, 2002), more specifically, they have been reported to elevate mRNA levels of GDF-11 in rat skeletal muscle (Suidasari et al., 2017).

Another component of one-carbon metabolism is the nutrient choline, which is an important molecule in transmethylation metabolic pathways. Choline reduces levels of homocysteine by converting it to methionine (Niculescu and Zeisel, 2002). Riboflavin, commonly known as vitamin B2, also demonstrates a protective effect as it is thought to protect brain tissue from oxidative ischemic distress (Mack et al., 1995). Furthermore, folic acid has been implicated in axonal regeneration after injury to the spinal and optic axons in vivo (Iskandar et al., 2010). Due to the potential of B-vitamins and choline in promoting neuroplasticity and poststroke recovery, it is beneficial to investigate the efficacy of these treatments for stroke. The purpose of this study is to investigate whether B-vitamin and choline supplementation increases plasticity after ischemic damage in a mouse model.

\section{MATERIALS AND METHODS}

\section{Animals}

All experiments were conducted in accordance with the Canadian Council of Animal Care. The experiments were performed in a randomized manner. At the age of one month, wildtype male mice were placed on either a control diet (CD, $2 \mathrm{mg} / \mathrm{kg}$ folic acid) or a folic acid deficient diet (FADD, $0.3 \mathrm{mg} / \mathrm{kg}$ folic acid) for four
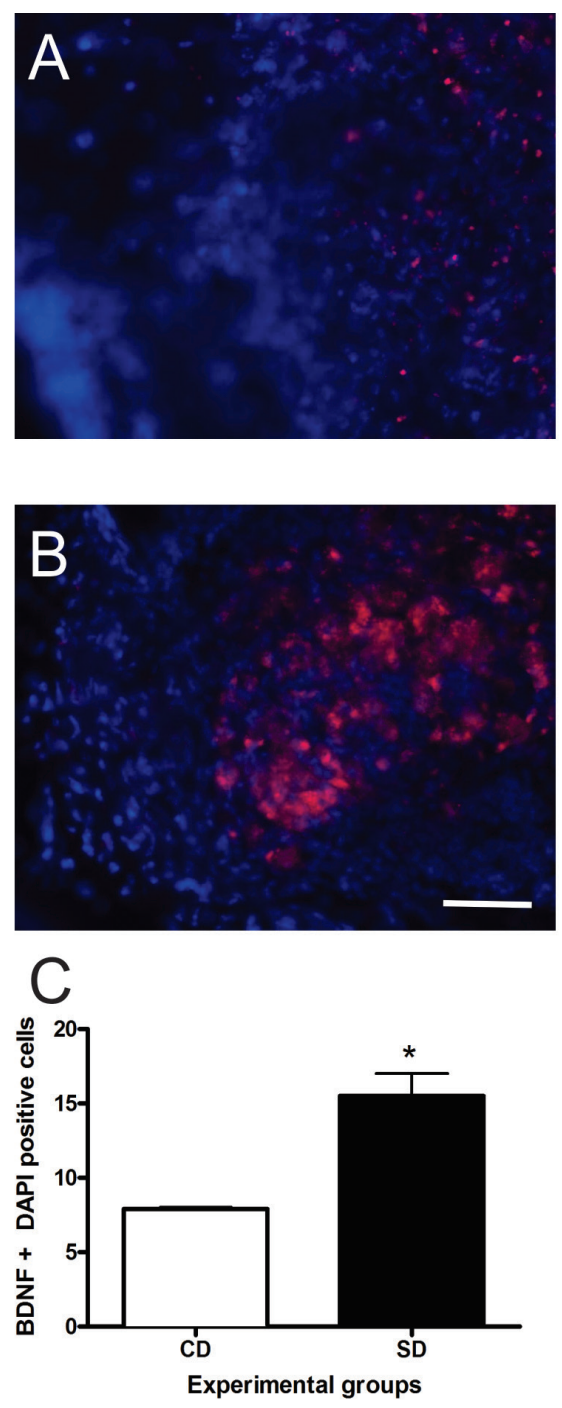

Figure 1. The impact of supplemental diet (SD) after ischemic damage on brain-derived neurotrophic factor (BDNF). Representative images of brain tissue stained with BDNF (red) and DAPI (blue) at the damage site from CD (A) and SD (B) mice. Quantification of BDNF and DAPI positive cells within the damage site $(C)$. $*$ indicates a $\mathrm{p}<0.05$. All pictures at 400X magnification, scale bar $=50 \mu \mathrm{m}$.

weeks preceding damage. Following damage, the FADD mice were placed on a supplemented diet (SD, $5 \mathrm{mg} / \mathrm{kg}$ folic acid) and the $\mathrm{CD}$ mice remained on the $\mathrm{CD}$. In addition to higher levels of folic acid, the SD contained increased levels of riboflavin $(10 \mathrm{mg} /$ $\mathrm{kg}$ diet; Agte et al., 1998), vitamin B12 (0.5 mg/kg diet; Wolff et al., 1998), and choline bitartrate (4950 mg/kg diet) compared to control diet. Levels of choline were based on previous studies (Craciunescu et al., 2003). In the present study, we used choline bitrate, and not choline chloride because of availability. The diets were custom-formulated by Envigo (Envigo, Madison, WI). The mice were maintained on the SD for four weeks following ischemic damage after which brain tissue was collected. 

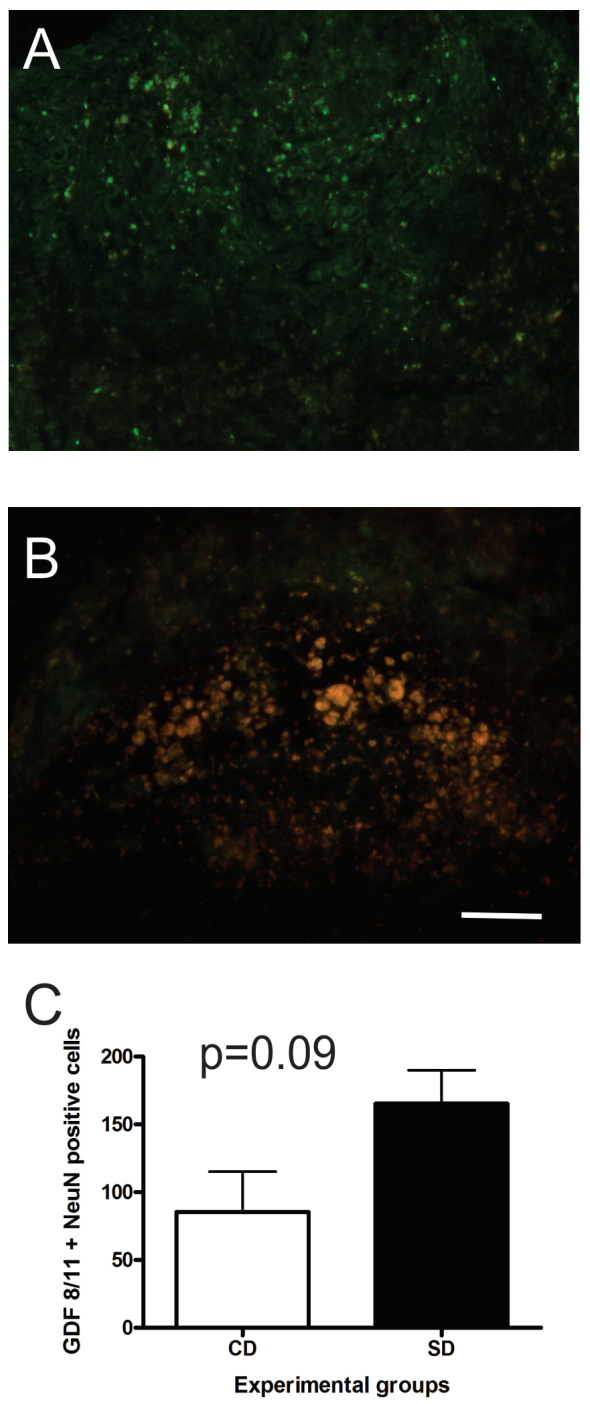

Figure 2. The impact of supplemental diet (SD) after ischemic damage on growth differential factor (GDF-8/110). Representative images of brain tissue stained with GDF-8/11 (red) and neuronal nuclei (NeuN; green) at the damage site from CD (A) and SD (B) mice. Quantification of GDF-8/11 and NeuN positive cells within the damage site (C). All pictures at 400X magnification, scale bar $=50 \mu \mathrm{m}$.

\section{Photothrombosis}

The procedure for photothrombosis was followed using the method previously described (Labat-gest and Tomasi, 2013). Briefly, the mice were anaesthetized with $3.5-4 \%$ isoflurane in an induction chamber. In preparation, the mouse head was shaved and disinfected, then moved to a stereotaxic frame. To maintain anesthesia, a face mask was used at $1.5-2 \%$ isoflurane. In addition, a temperature probe and heating pad was used to maintain body temperature at $36^{\circ} \mathrm{C}$. Five minutes before exposure to the laser Rose Bengal was injected intraperitoneally. To induce ischemia, a $532 \mathrm{~nm}$ laser light was placed and shone $2 \mathrm{~cm}$ above the sensorimotor cortex for $15 \mathrm{~min}$. Afterwards, animals were returned to their home cages.

\section{Analysis of Brain Tissue}

After ischemic damage was induced using photothrombosis, brain tissue from four mice per group was sectioned (30 $\mu \mathrm{m}$ thick) and stained with anti-BDNF (BDNF, 1:100) (AbCam, Cambridge, MA), or anti-GDF-8+11 (1:100) (AbCam, Cambridge, MA). All brain tissue was co-stained with DAPI $(1: 10,000)$ (ThermoFisher, Waltham, MA), a nuclear stain, or anti-neuronal nuclei (NeuN, 1:200) (AbCam, Cambridge, MA), for neuronal nuclei quantification (Gusel'nikova and Korzhevskiy, n.d.; Tarnowski et al., 1991). Staining was visualized using a microscope (Zeiss) and all images were collected at 40X magnification. Within the damage site, ImageJ (Rueden et al., 2017) was used to quantify incidence of colocalization of BDNF with DAPI and GDF-11 and NeuN cells in both $\mathrm{CD}$ and $\mathrm{SD}$ brain tissue.

\section{Statistics}

Data analysis was performed by two individuals blinded to experimental groups. Statistical analysis was performed using GraphPad Prism 6.0 (GraphPad Software, La Jolla, CA). Unpaired t-tests were used to compare the means between SD and CD mice. All data are presented as mean \pm standard error of the mean (SEM). Statistical tests were performed using a significance level of 0.05 .

\section{RESULTS}

Approximately 30 days after photothrombosis, analysis of the total BDNF co-localized with DAPI took place. Within the lesion area, mice on the SD had more BDNF positive cells compared to the $\mathrm{CD}$ animals (Figure 1; $t(3)=5.05, p<0.05$ ). Total BDNF levels include levels in both neurons and astrocytes. Additionally, we observed a trend for increased levels of GDF-8/11 co-localized with NeuN cells of SD mice compared to those of CD mice within the damage area (Figure 2; $t(3)=2.09, p=0.09$ ).

\section{DISCUSSION}

In this study, we found that mice maintained on a SD after ischemic damage had increased levels of BDNF within neurons and astrocytes at the damage site compared to $\mathrm{CD}$ mice. Interestingly, there was also a trend for increased levels of neuronal GDF-8/11 within the damage site of SD mice compared to CD mice.

The photothrombotic mouse model is reproducible and used to study ischemic damage due to the well-defined ischemic regions produced and rapid progression of ischemic cell death (Dietrich et al., 1986; Fluri, 2015; Watson et al., 1985). Yet, a limitation of the photothrombotic model in accurately mimicking human stroke as strokes are typically caused by blood flow interruption (Labat-gest and Tomasi, 2013). Additionally, in some stroke patients, spontaneous reperfusion may cause secondary damages, which cannot be investigated using this model (Labat-gest and Tomasi, 2013). Nonetheless, this model is useful in the study of plasticity, as it allows processes in the perilesional area to be studied and aids in the investigation of neuronal repair following stroke (Kleinschnitz et al., 2015).

The results from our study suggest that the effects of folic acid on post-stroke neuroplasticity in mice are mediated by BDNF. The 
data demonstrates that B-vitamins, including folic acid, riboflavin and vitamin B12, as well as choline supplementation can influence neuroplasticity in the adult central nervous system, indicating that these effects are not confined to the embryonic period. This aligns with the outcome of previous experiments on the effects of folic acid supplementation on axonal regeneration in the adult mouse spinal cord (Guseva et al., 2008; Iskandar et al., 2010). Elevated levels of GDF-11 may also demonstrate a neuroprotective effect. Brain GDF-11 levels decline after stroke and with age (Chauhan et al., 2016). However, supplementation with rGDF-11 has been reported to improve post-stroke injury in mice, suggesting that increasing dietary levels of B vitamins and choline may exhibit neuroprotective effects due to elevation of GDF-11 levels (Chauhan et al., 2016). Additionally, GDF-11 may protect against endothelial injury through an anti-inflammatory effect, contributing to improved stroke recovery (Mei et al., 2016).

The mechanism by which BDNF levels are increased through B-vitamin and choline dietary supplementation must be further explored. A study on the neuroblastoma cell line found that H-89 suppressed the neuroprotective effects of folic acid against dexamethasone-induced neurotoxicity in mice (Budni et al., 2011). A competitive inhibitor of protein kinase A (PKA), H-89 competes with ATP for binding to the catalytic subunit (Chijiwa et al., 1990). In turn, PKA is involved in various physiological functions in the brain, including neurotransmitter release, gene expression, synaptic plasticity, memory, cell growth and differentiation, and cell survival (Xue et al., 2016). These PKA-mediated functions occur through the phosphorylation of substrates such as the cAMP responsive element binding protein (CREB), which is known to cause the expression of BDNF upon activation (Xue et al., 2016). Thus, BDNF levels may be raised in mice fed folic acid-supplemented diets through the activation of PKA. Additional mechanisms behind stroke rehabilitation include the link between folic acid and reduced oxidative stress as well as prevention of neurotoxicity (Cagnacci et al., 2015; Lipton et al., 1997). Folic acid has been reported to prevent neurotoxicity induced by glutamate and the glutamate receptor NMDA in cultured mouse cerebellar granule neurons (Lin et al., 2004). In an axonal regeneration study, folate-mediated regeneration was dependent on the induction of the folate receptor alpha (Folr1). Therefore, it is necessary to further investigate the mechanisms by which Folr1 encourages axonal regeneration (Iskandar et al., 2010). Riboflavin may also exert its neuroprotective effect by raising the levels of BDNF, as a riboflavin-supplemented diet was found to increase BDNF levels in a mice model of multiple sclerosis (Naghashpour et al., 2016). Interestingly, vitamin B12 may not contribute to higher levels of BDNF since vitamin B12 supplementation has shown lower cortex BDNF levels compared to those of a control group (Rathod et al., 2016). However, vitamin B12 and omega-3 fatty acid supplementation was found to increase levels of the neurotrophin nerve growth factor (NGF), suggesting that NGF is a potential target molecule for analysis in further studies on B vitamin supplementation, neuroplasticity, and stroke (Rathod et al., 2016). The actions of choline in the supplemented diet may be similar to those of other B vitamins participating in one-carbon metabolism, thus lowering serum levels of homocysteine (Niculescu and Zeisel, 2002). In folate-deficient individuals, more methyl groups are used from choline in methylation of homocysteine (Niculescu and Zeisel, 2002). Choline might also provide protection after ischemic stroke by facilitating angiogenesis (Jin et al., 2015).

Much of the current literature focuses on how folic acid can lower stroke risk, but few studies investigate at the relationship between $\mathrm{B}$ vitamins and choline with post-stroke rehabilitation. The use of folic acid and other B vitamins as a therapy is promising, and its safety makes it a valuable alternative treatment for rehabilitation. While the results from this experiment are significant in showing the relationship between dietary supplementation of B-vitamins and choline on post-stroke neuroplasticity, there are further areas of research that must be explored. When recovering, many human patients make rapid gains in the first few weeks following ischemia, only to have their recovery plateau (Corbett et al., 2015). Studies have shown that there is a critical period for recovery during which therapies are most effective when applied (Corbett et al., 2015). It is important to understand the window during which the optimal effects of B vitamin and choline supplemented therapies may be realized. It is also important to investigate the ideal dosage for post-stroke plasticity. In a study of mice investigating the effects of folic acid on dexamethasone-induced neurotoxicity, maximum protection was achieved at a concentration of $300 \mu \mathrm{M}$ of folic acid, resulting in a 29\% protection rate (Budni et al., 2011). In another study, folic acid applied at a concentration of $80 \mu \mathrm{g} / \mathrm{kg}$ had the greatest effect on regeneration in the adult nervous system (Iskandar et al., 2010). Accordingly, these both serve as potential starting points that can be used in future studies investigating the ideal dosage concentration for treatments.

Future studies should investigate the efficacy of dietary supplementation with B vitamins and choline in humans affected by stroke. Many results observed in mouse models have not translated onto human models due to humans being unable to safely consume the same dosage as mice. Therefore, results from the present study must be approached with caution (Ulrich Dirnagl, 2006). However, since B vitamins are safe for consumption, this may no longer be an issue. The results demonstrate promise, highlighting a safe, yet effective therapy for recovery following stroke. Combining nutritional changes with other therapies for stroke-affected individuals may be a highly effective treatment.

\section{ACKNOWLEDGMENTS}

PK would like to thank the Ontario Online Research CO-OP for the opportunity to do research and Allen Flemington for guidance throughout the project. NMJ is supported by fellowships from Fonds de la recherché en santé Québec (FRSQ), the Council of Ontario Universities Women's Health Scholars and Natural Sciences and Engineering Research Council.

\section{REFERENCES}


Agte VV, Paknikar K, Chiplonkar SA. (1998). Effect of riboflavin supplementation on zinc and iron absorption and growth performance in mice. Biological Trace Element Research, 65(2), 109-115. doi:10.1007/BF02784263

Brun CE and Rudnicki MA. (2015). GDF11 and the Mythical Fountain of Youth. Cell Metabolism, 22(1), 54-56. doi:10.1016/j.cmet.2015.05.009

Budni J, Romero A, Molz S, Martín-de-Saavedra MD, Egea J, Del Barrio L, Tasca CI, Rodrigues AL, López MG. (2011). Neurotoxicity induced by dexamethasone in the human neuroblastoma SH-SY5Y cell line can be prevented by folic acid. Neuroscience, 190, 346-353. doi:10.1016/j.neuroscience.2011.05.053

Cagnacci A, Cannoletta M, Xholli A, Piacenti I, Palma F, Palmieri B. (2015). Folate administration decreases oxidative status and blood pressure in postmenopausal women. European Journal of Nutrition, 54(3), 429-435. doi:10.1007/ s00394-014-0726-8

Castro R, Rivera I, Blom HJ, Jakobs C, Tavares de Almeida I. (2006). Homocysteine metabolism, hyperhomocysteinaemia and vascular disease: an overview. Journal of Inherited Metabolic Disease, 29(1), 3-20. doi:10.1007/ s10545-006-0106-5

Chauhan A, Hudobenko J, Patrizz A, McCullough LD. (2016). Abstract 65: Restoration of Growth Differentiation Factor 11 Protects Against Stroke in Mice. Stroke, 47(Suppl 1), A65 LP-A65.

Chen A, Xiong L-J, Tong Y, Mao M. (2013). The neuroprotective roles of BDNF in hypoxic ischemic brain injury. Biomedical Reports, 1(2), 167-176. doi:10.3892/br.2012.48

Chijiwa T, Mishima A, Hagiwara M, Sano M, Hayashi K, Inoue T, Naito K, Toshioka T, Hidaka H. (1990). Inhibition of forskolin-induced neurite outgrowth and protein phosphorylation by a newly synthesized selective inhibitor of cyclic AMP-dependent protein kinase, N-[2-(p-bromocinnamylamino)ethyl]5-isoquinolinesulfonamide (H-89), of PC12D pheochromocytoma cells. The Journal of Biological Chemistry, 265(9), 5267-72.

Cohen-Cory S, Kidane AH, Shirkey NJ, Marshak S. (2010). Brain-derived neurotrophic factor and the development of structural neuronal connectivity. Developmental Neurobiology, 70(5), 271-288. doi:10.1002/dneu.20774

Corbett D, Jeffers M, Nguemeni C, Gomez-Smith M, Livingston-Thomas J. (2015). Lost in translation. In Progress in brain research (Vol. 218, pp. 413 434). doi:10.1016/bs.pbr.2014.12.002

Craciunescu CN, Albright CD, Mar M, Song J, Zeisel SH. (2003). Choline Availability During Embryonic Development Alters Progenitor Cell Mitosis in Developing Mouse Hippocampus. The Journal of Nutrition, 133(18), 3614 3618.

Crozier RA, Bi C, Han YR, Plummer MR. (2008). BDNF Modulation of NMDA Receptors Is Activity Dependent. Journal of Neurophysiology, 100(6), 3264 3274. doi:10.1152/jn.90418.2008

Dennis KE and Levitt P. (2005). Regional expression of brain derived neurotrophic factor (BDNF) is correlated with dynamic patterns of promoter methylation in the developing mouse forebrain. Molecular Brain Research, 140(1-2), 1-9. doi:10.1016/j.molbrainres.2005.06.014

Dietrich WD, Ginsberg MD, Busto R, Watson BD. (1986). Photochemically Induced Cortical Infarction in the Rat. 1. Time Course of Hemodynamic Consequences. Journal of Cerebral Blood Flow \& Metabolism, 6(2), 184-194. doi: $10.1038 /$ jcbfm. 1986.31

Dirnagl U. (2006). Bench to bedside: the quest for quality in experimental stroke research. Journal of Cerebral Blood Flow and Metabolism, 26(12), 1465 1478. doi:10.1038/sj.jcbfm.9600298

Dirnagl U, Iadecola C, Moskowitz MA. (1999). Pathobiology of ischaemic stroke: an integrated view. Trends in Neurosciences, 22(9), 391-7.

Fluri F. (2015). Animal models of ischemic stroke and their application in clinical research. Drug Design, Development and Therapy, (9), 3445-3454.

Gusel'nikova VV and Korzhevskiy DE. (2015). NeuN As a Neuronal Nuclear Antigen and Neuron Differentiation Marker. Acta Naturae, 7(2), 42-7.

Guseva MV, Hopkins DM, Scheff SW, Pauly JR. (2008). Dietary Choline Supplementation Improves Behavioral, Histological, and Neurochemical Outcomes in a Rat Model of Traumatic Brain Injury. Journal of Neurotrauma, 25(8), 975-983. doi:10.1089/neu.2008.0516

Hara Y. (2015). Brain Plasticity and Rehabilitation in Stroke Patients. Journal of Nippon Medical School, 82(1), 4-13. doi:10.1272/jnms.82.4

Horbelt D, Denkis A, Knaus P. (2012). A portrait of Transforming Growth Factor $\beta$ superfamily signalling: Background matters. International Journal of Biochemistry \& Cell Biology, 44(3), 469-74. doi:10.1016/j.biocel.2011.12.013

Huo Y, Li J, Qin X, Huang Y, Wang X, Gottesman RF, Tang G, Wang B, Chen D, He M, Fu J, Cai Y, Shi X, Zhang Y, Cui Y, Sun N, Li X, Cheng X, Wang J, Yang X, Yang T, Xiao C, Zhao G, Dong Q, Zhu D, Wang X, Ge J, Zhao L, Hu D, Liu L, Hou FF, and CSPPT Investigators. (2015). Efficacy of Folic Acid Therapy in Primary Prevention of Stroke Among Adults With Hypertension in China. Journal of the American Medical Association, 313(13), 1325. doi:10.1001/jama.2015.2274

Iskandar BJ, Rizk E, Meier B, Hariharan N, Bottiglieri T, Finnell RH, Jarrad DF, Banerjee RV, Skene JH, Nelson A, Patel N, Gherasim C, Simon K, Cook TD, Hogan KJ. (2010). Folate regulation of axonal regeneration in the rodent central nervous system through DNA methylation. Journal of Clinical Investigation, 120(5), 1603-1616. doi:10.1172/JCI40000

Jiang Y, Wei N, Lu T, Zhu J, Xu G, Liu X. (2011). Intranasal brain-derived neurotrophic factor protects brain from ischemic insult via modulating local inflammation in rats. Neuroscience, 172, 398-405. doi:10.1016/j.neuroscience.2010.10.054

Jin X, Wang R, Wang H, Long C, Wang H. (2015). Brain protection against ischemic stroke using choline as a new molecular bypass treatment. Acta Pharmacologica Sinica, 36(12), 1416-1425. doi:10.1038/aps.2015.104

Katsimpardi L, Litterman NK, Schein PA, Miller CM, Loffredo FS, Wojtkiewicz GR, Chen JW, Lee RT, Wagers AJ, Rubin LL. (2014). Vascular and neurogenic rejuvenation of the aging mouse brain by young systemic factors. Science, 344(6184), 630-4. doi:10.1126/science.1251141

Kleinschnitz C, Fluri F, Schuhmann M. (2015). Animal models of ischemic stroke and their application in clinical research. Drug Design, Development and Therapy, 9, 3445. doi:10.2147/DDDT.S56071

Labat-gest V Tomasi S. (2013). Photothrombotic ischemia: a minimally invasive and reproducible photochemical cortical lesion model for mouse stroke studies. Journal of Visualized Experiments, (76). doi:10.3791/50370

Lin Y, Desbois A, Jiang S, Hou ST. (2004). Group B vitamins protect murine cerebellar granule cells from glutamate/NMDA toxicity. Neuroreport, 15(14), 2241-4.

Lipton SA, Kim WK, Choi YB, Kumar S, D’Emilia DM, Rayudu PV, Arnelle DR, Stamler JS. (1997). Neurotoxicity associated with dual actions of homocysteine at the N-methyl-D-aspartate receptor. Proceedings of the National Academy of Sciences of the United States of America, 94(11), 5923-8.

Mack CP, Hultquist DE, Shlafer M. (1995). Myocardial Flavin Reductase and Riboflavin: A Potential Role in Decreasing Reoxygenation Injury. Biochemical and Biophysical Research Communications, 212(1), 35-40. doi:10.1006/ bbrc.1995.1932

Mei W, Xiang G, Li Y, Li H, Xiang L, Lu J, Xiang L, Dong J, Liu M. (2016). GDF11 Protects against Endothelial Injury and Reduces Atherosclerotic Lesion Formation in Apolipoprotein E-Null Mice. The Journal of the American Society of Gene Therapy, 24(11), 1926-1938. doi:10.1038/mt.2016.160

Murphy T, Dias GP, Thuret S. (2014). Effects of diet on brain plasticity in animal and human studies: mind the gap. Neural Plasticity, 563160. doi:10.1155/2014/563160

Naghashpour M, Amani R, Sarkaki A, Ghadiri A, Samarbafzadeh A, Jafarirad S, Malehi AS. (2016). Brain-derived neurotrophic and immunologic factors: beneficial effects of riboflavin on motor disability in murine model of multiple sclerosis. Iranian Journal of Basic Medical Sciences, 19(4), 439-48.

Niculescu MD and Zeisel SH. (2002). Diet, Methyl Donors and DNA Methylation: Interactions between Dietary Folate, Methionine and Choline. The Journal of Nutrition, 132(8), 2333S-2335S. doi:10.1093/jn/132.8.2333S

Rathod RS, Khaire AA, Kale AA, Joshi SR. (2016). Effect of vitamin B12 and omega-3 fatty acid supplementation on brain neurotrophins and cognition in rats: A multigeneration study. Biochimie, 128-129, 201-208. doi:10.1016/j. biochi.2016.08.009

Rueden CT, Schindelin J, Hiner MC, DeZonia BE, Walter AE, Arena ET, Eliceiri KW. (2017). ImageJ2: ImageJ for the next generation of scientific image data. BMC Bioinformatics, 18(1), 529. doi:10.1186/s12859-017-1934-Z

Sable P, Dangat K, Kale A, Joshi S. (2011). Altered brain neurotrophins at birth: consequence of imbalance in maternal folic acid and vitamin B12 metabolism. Neuroscience, 190, 127-134. doi:10.1016/j.neuroscience.2011.05.010 
Selhub J. (2002). Folate, vitamin B12 and vitamin B6 and one carbon metabolism. The Journal of Nutrition, Health \& Aging, 6(1), 39-42.

Suidasari S, Uragami S, Yanaka N, Kato N. (2017). Dietary vitamin B6 modulates the gene expression of myokines, Nrf2-related factors, myogenin and HSP60 in the skeletal muscle of rats. Experimental and Therapeutic Medicine, 14(4), 3239-3246. doi:10.3892/etm.2017.4879

Tarnowski BI, Spinale FG, Nicholson JH. (1991). DAPI as a useful stain for nuclear quantitation. Biotechnic \& Histochemistry, 66(6), 297-302.

Watson BD, Dietrich WD, Busto R, Wachtel MS, Ginsberg MD. (1985). Induction of reproducible brain infarction by photochemically initiated thrombosis. Annals of Neurology, 17(5), 497-504. doi:10.1002/ana.410170513

Wolff GL, Kodell RL, Moore SR, Cooney CA. (1998). Maternal epigenetics and methyl supplements affect agouti gene expression in Avy/a mice. The FASEB Journal, 12(11), 949-957.

Xue W, Wang W, Gong T, Zhang H, Tao W, Xue L, Sun Y, Wang F, Chen G. (2016). PKA-CREB-BDNF signaling regulated long lasting antidepressant activities of Yueju but not ketamine. Scientific Reports, 6(1), 26331. doi:10.1038/ srep26331

Zhang J, Yu Z, Yu Z, Yang Z, Zhao H, Liu L, Zhao J. (2011). rAAV-mediated delivery of brain-derived neurotrophic factor promotes neurite outgrowth and protects neurodegeneration in focal ischemic model. International Journal of Clinical and Experimental Pathology, 4(5), 496-504.

Zhang M, Jadavji NM, Yoo HS, Smith PD. (2018). Recombinant growth differentiation factor 11 influences short-term memory and enhances Sox 2 expression in middle-aged mice. Behavioural Brain Research, 341(July 2017), 45-49. doi:10.1016/j.bbr.2017.12.019 